\title{
Recurrent Pneumonia Caused by Genetic Immunodeficiency: A Prophylactic and Rehabilitative Approach
}

Renata Cristina de Angelo Calsaverini Leal ${ }^{1}$, Érika Cristina Pavarino Bertelli ${ }^{2}$ and Zaida Aurora Sperli Geraldes Soler ${ }^{3}$ ${ }^{I}$ Student of the Post-Graduation Course (Master's degree Program in Medicine and Correlated Sciences) of the São José do Rio Preto School of Medicine (FAMERP); ${ }^{2}$ Adjunct Professor at the Department of Molecular Biology of the São José do Rio Preto School of Medicine (FAMERP);

${ }^{3}$ Adjunct Professor, Social and Community Services Director of the São José do Rio Preto School of Medicine (FAMERP); São José do Rio Preto; SP, Brazil

\begin{abstract}
Recurrent infections are a consequence of a series of genetic diseases characterized by deficiency in the immunological response. One of these diseases is the agammaglobulinemia, which is characterized by the basic defect in the maturation of lymphocytes B. The carrier of this kind of immunodeficiency, which is linked to the X (XLA) chromosome, has had primary pneumonias that have evolved into secondary pneumonias (chronic lungs with sequelae) after the third or fourth year of life. The clinical and rehabilitative quest for prophylaxis against the XLA immunodeficiency is accomplished in order to avoid the evolution of the bacterial infection into sequelae and loss of pulmonary function, which propitiates the recurrence of the disease and deteriorates the life quality of the patient. Forty cases of recurrent respiratory infections were studied. Some of them were associated with primary respiratory diseases without investigation of serum immunoglobulins and some were not. Casuistics was performed according to data from medical records with pertinent treatments collected from January 1997 to September 2004 at the Specialized Physiotherapy Center. Age average was 2.7 years of life. It is statistically impossible to precise results concerning only the immunosuppressed patients due to the lack of specific diagnosis. That is explained by the fact that recurrent XLA pneumonias may be attributed to the gastroesophageal reflux disease or to bronchial asthma. However, the improved results showed by the pulmonary function as preventive strategy were attributed to the respiratory physiotherapy, since intravenous immunoglobulin replacement therapies were not performed. Respiratory physiotherapy acts as a supportive factor in the healing process and occupies a fundamental role in the prophylaxis against recurrent respiratory clinical features, especially those of obstructive and secretionary characteristics.

Key-Words: Pneumonia, immunodeficiency, physiotherapy.
\end{abstract}

The immune system, composed by trillions of cells and genetically characterized by the somatic rearrangement that generates diversity to deal with a great variety of intruder microorganisms, has a high degree of precision. However, it may leave lacunas. These are the genetic anomalies, more specifically, the immunodeficiency primary diseases [1].

The bone marrow produces the lymphocytes, also known as precursor cells. Their maturation makes it possible to secrete antibodies, initiating the humoral phase of the immune system, which is considered the third way of attack against an antigen. Another important role of the B cells, which are originated in the lymphoid organs, is to product T-lymphocytes, whose differentiation occurs in the thymus [2].

In the humoral immune response, exogenous particles are presented together with MHC class II molecules (antigenpresenting cells). The antigens do not act directly on the nonactivated B lymphocytes. Instead, they link themselves to the histocompatibility cells (MHC class II). These cells englobe the antigens, which are partially digested inside the endosomes

Received on 15 January 2007; revised 8 June 2007.

Address for correspondence: Dr. Renata Cristina de Angelo Calsaverini Leal. E-mail: ar.1eal@uol.com.br. Study accomplished at the Department of Genetics of the São José do Rio Preto School of Medicine and at the Specialized Physiotherapy Center in Tanabi SP, Brazil.

The Brazilian Journal of Infectious Diseases 2007;11(3):307-310. (C) 2007 by The Brazilian Journal of Infectious Diseases and Contexto Publishing. All rights reserved. (digestive organelles). The resulting peptides containing amino acids associate themselves to the histocompatibility cells. These peptides derived from the antigens and associated to the class I MHC cells (T lymphocyte activator) or class II MHC cells (B lymphocyte activator), result in the interaction of the latter with the lymphocytes. After this process, the phagocyted antigen is presented to another type of lymphocyte: the 'helper' (Th) lymphocytes, which stimulate B lymphocytes (genic transcription) into producing antibodies (clonal activation) [3].

Each plasmocyte is capable of secreting approximately 10 million antibody molecules per hour during a period up to 7 days. Superposed to the great number of molecules there is still the variations in the DNA sequences, whose immunoglobulins (antibodies) acquire binding characteristics with high affinity level to the microorganisms due to the form of its protein. The production of antibodies accomplished by mature B lymphocytes is, in short, the specialty of the somatic defense. In the presence of the first contact with a pathogenic agent, the system produces the IgM as the first antibody to act, being followed by the IgG [4].

The antibodies (immunoglobulins) link themselves to the invader agent or to the toxin produced by him, facilitating the phagocytosis or neutralizing its toxic action, respectively. Amounted to the immunoglobulins genic variability, there is also the lymphocytes somatic mutation. The macrophages, neutrophils and T-lymphocytes complete the phagocytosis process through their interaction with the immunoglobulins [5]. 
Like all proteins, the antibodies are genetically encoded. They are constituted of two chains, named L (light) and $\mathrm{H}$ (heavy), interconnected by bridges. In each of the chains referred above there are two regions: the constant (c), which determines the class of the Ig protein, and the variable (v), which translates its specificity and forms the binding site of the antibody through its amino acid extremity.

Each immunoglobulin has a specific function:

- $\mathrm{IgG}$ - responsible for fighting off infections; goes through the placenta, therefore conferring passive immunity to the fetus and the neonate.

- $\operatorname{IgM}$ - first antibody formed in response to an antigen; more effective than $\mathrm{IgG}$ in the fixation of the amino complement.

- $\operatorname{Ig} \mathrm{A}$ - especially important in the secretions; protects the body surfaces and defends the intestine against microorganisms.

- $\operatorname{IgD}$ - obscure.

- $\operatorname{IgE}$ - involved in allergic reactions and in histamine liberation; can fight off intestinal parasites [2].

Hereditary immunodeficiency may be linked to a single gene (monogenic disorder of the immune system), provoking disastrous chain situations.

Recurrent infections are a consequence of a series of genetic diseases characterized by deficiency in the immunological response. One of these diseases is the agammaglobulinemia with X (XLA)-linked inheritance, which occurs due a lack of plasmocyte. This basic defect in the maturation of the type B leukocytes detains the gravity in the absence of the immunoglobulins: the specific proteins of the somatic defense. The non-maturation of the B lymphocytes, which is characteristic of this anomaly, is caused by the defect of the kinase protein [6].

Studies confer survival of patients with agammaglobulinemia due to small concentrations of transplacentary $\operatorname{IgG}$ and immunity mediated by $\mathrm{T}$ lymphocytes, more specifically the toxic cells. These cells emit a chemical substance that destroys the infected cell through its link with the peptide-MHC complex. The passive immunity lasts for a period up to six months, not only to the immunodeficiency carriers but also to healthy babies, who start to produce their own antibodies at that age, even if the way they do it is still inadequate (hypoagammaglobulinemia) [1].

The agammaglobulinemia genetic defect includes the XLA in the group of the primary immunodeficiency diseases due to mutation of the BTK gene. It makes the patient susceptible to recurrent bacterial infections mainly caused by Streptococcus pneumoniae (sp.) and Haemophilus influenzae [7].

Considered the main etiological agent of pneumoniae in general, among the community as well as among the nosocomial cases, the sp., despite being Gram-positive bacteria, confers its virulence according to the quantity of polysaccharides contained in its capsule and, especially, to the immunity reaction it causes. To the XLA, the sp. transforms itself into a superantigen, not only because of its enhanced virulence in the carrier, but also because of the absence of memory cells, what makes it possible for eventual infections to be even more devastating [8].

The clinical quest for prophylaxis against the XLA immunodeficiency is accomplished in order to avoid that the pulmonary bacterial infection transforms itself from primary (previously healthy lungs) into secondary (lungs with previous diseases such as bronchiectasias, emphysema, ciliary dyskinesia, chronic bronchitis, pulmonary consequences of gastroesophageal reflux and bronchial dysplasia) irremediably caused by recurrent infections of the infiltrated type. The persistent cough and sibilance associated to the toxemia, and aggravated by the infections caused by highly pathogenic microorganisms, includes frequency and duration of these clinical features. Other findings such as suppurated medium otitis, prolonged maxillary sinusitis, and poliarthritis lead to the diagnosis of the primary immunodeficiency, which is confirmed by laboratorial tests [9].

Forty cases of recurrent respiratory infections were investigated in a retrospective study. Some of them were associated with primary respiratory diseases without investigation of serum immunoglobulins and some were not. Casuistics was performed according to data from medical records with pertinent treatments, and collected from January 1997 to September 2004 at the Specialized Physiotherapy Center. Age average was 2.7 years of life.

All patients in infectious phase were under clinical antimicrobial treatment. Those in the intercrisis phase were making use of medications to control other associated pathologies such as: gastroesophageal reflux disease and bronchial asthma.

The physiotherapeutic intervention happened during the entire period of infectious exacerbation and there were distinct groups who complied with the treatment during the intercrisis phase.

First symptoms of asthmatiform chronicity related to the airflow limitation due to bronchial remodeling were found to appear at the age of 4 (90\% of the group). Another group (65\%) composed by patients with pulmonary sequelae, presented tracheobronchial hypersecretion associated to paranasal sinus pathology as a consequence of dynamical GER caused by persistent cough. A small number of these patients (2\%) might have developed a greater risk of pulmonary infection due to signs of bronchiectasis.

Physiotherapeutic resources maintain the bronchial permeability, avoid the culture environment and optimize the pulmonary functionality [10].

Although the fact that the antimicrobial treatment based on the sputum culture responds better to diagnostic and therapeutic purposes, the choice for a precocious treatment usually seems to be a consensus among doctors. They have in mind the pneumoniae diagnosed as Gram-positive due to the classical form of the disease. This decision is reinforced by the inconvenient lateness of laboratorial results in isolating the etiological agent. The bacterial isolation would be 
unnecessary for lower airway infections of less gravity, since the beta-hemolitic streptococci, Haemophilus influenzae and Staphylococcus aureus are present in the microbial flora in the nasopharynges. However, this is a counterpoint to the primary immunodeficiency cases, given the fact that the differential clinical diagnosis is the gravity of the pneumonia and its recurrence. This is explained by the fact that normal infants have a characteristic difficulty in producing antibodies against polysaccharide antigens. This fact justifies the great frequency of infection by Haemophilus influenzae during the early infancy $[8,9]$.

The great majority of the patients with lower airway infection are treated at home. Therefore it is easy to realize the fundamental role of physiotherapy when dealing with the disease.

Coughing is a fundamental tool in the rehabilitation process, not only for the physiodiagnosis but also for making the choice concerning therapeutic resources. In the presence of pleural effusion and other associated morbid factors, coughing should be stimulated with precaution, but never inhibited. In non-adverse situations, coughing may be inhibited by pain or reflex deficiency. Parenchymatous lesions of the infiltrated or diffuse type or lobar condensations should always be treated with intense and vigorous physiotherapy in order to accomplish the mobilization of mucus and propitiate alveolar reexpansion. In clinical features of lobar condensation, in special, the physiotherapeutic practice should be intensive and localized. The physiotherapist should associate the intensive airway clearance therapy to localized reexpansion resources and to kinesiotherapy of respiratory functional reeducation [10].

Figure 1. Study of patients with recurrent respiratory infection. Average Age 2.7 years. Period: from January 1997 to September 2004.
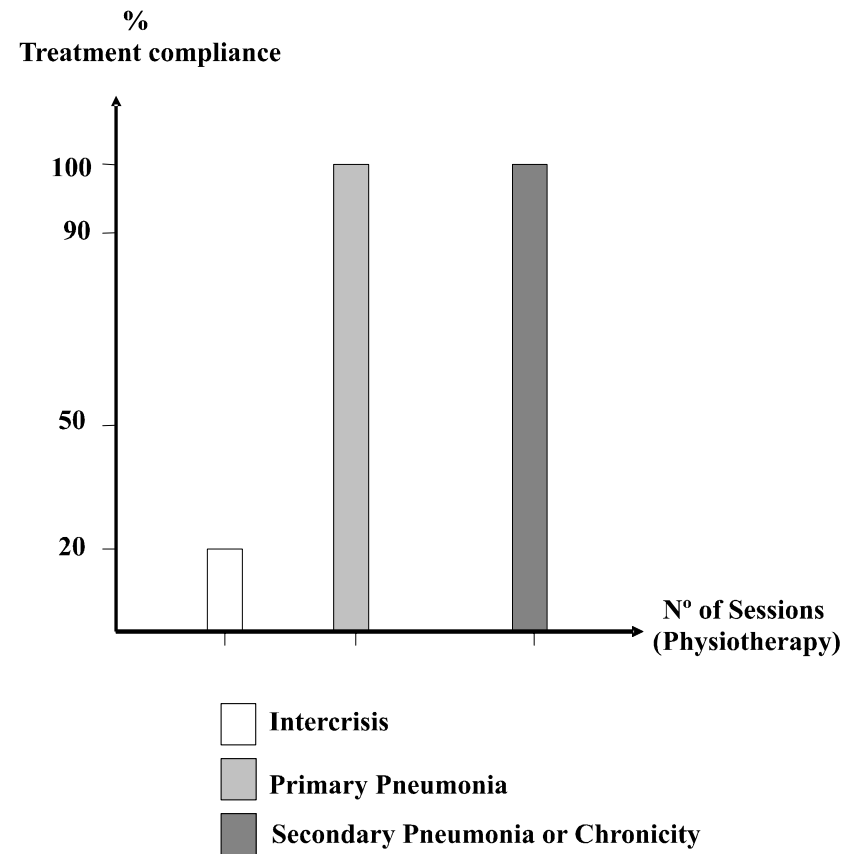

The XLA carrier may keep primary pneumoniae up to his/ her $3^{\text {rd }}$ or $4^{\text {th }}$ year of life, then evolving to secondary pneumoniae (chronic lungs with sequelae). Thus the systematic physiotherapeutic intervention aims to maintain the respiratory system of these patients in normal physiological conditions during the exacerbation intermissions [11].

The accomplished study revealed a $100 \%$ compliance with physiotherapy and functional reeducation in a systematic way of patients whose lungs presented sequelae and for all in the infectious phase. As for those in the intercrisis period there was a $20 \%$ statistics (Figure 1). For this group there was a result of rapid perfusional optimization: for those who were submitted to physiotherapy before the age of four years there was an increase from $30 \%$ to $60 \%$ in the intermission of the crisis. Also, there was an improvement of the pulmonary function for those with permanent or seasonal airflow limitation, hypersecretion and hyperreactivity with less severe exacerbations happening during longer intercrisis intermissions. As for those with primary pneumonia and preserved pulmonary function, a permeable and ventilated respiratory system was evinced after 10 days of physiotherapy and antimicrobial therapy (Figure 2).

In general, recurrent pneumoniae for patients who are hypersensitive to climatic and environmental exposure have revealed fully satisfactory results concerning the acute infectious clinical feature. Thus, the gravity of the second episode decreases considerably. The assertive intervention considerably increases the exacerbation intermissions $[10,11]$.

In patients with situation of instability of the immune and respiratory functional system, physiotherapy includes itself

Figure 2. Results of the study with patients from the Specialized Physiotherapy Center in the period from January 1997 to September 2004. Age Average: 4 years.

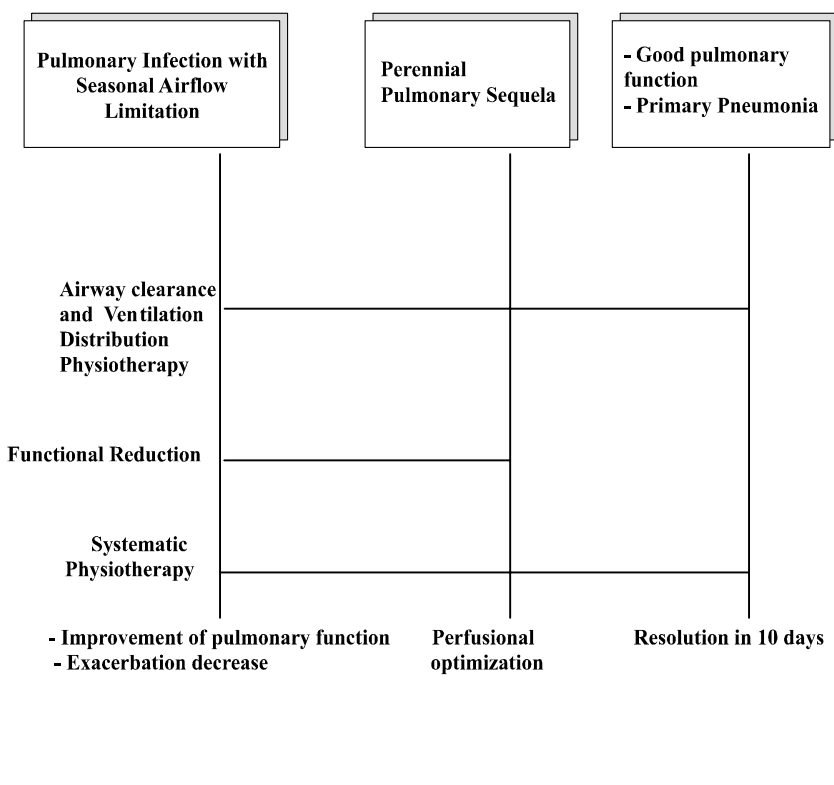


as a therapeutic indication. The physiotherapist is a member of the multiprofessional team and his role is to reestablish the pulmonary function.

Rehabilitation, in a general, depends on the patient complying with the treatment in a systematic way. Thus, the sample was fully considered in the analysis of the results, given the fact that treatment compliance was of $100 \%$. These patients are presented in a mixed way, since the congenit immunodeficiency ends up fusing itself to other pathologies. That happens not only from the diagnostic perspective but also from the evolutionary one. Therefore, we have the followup of patients with recurrent pneumonia originated from XLA, physiological hypoagammaglobulinemia, GER, and asthma. There were all treated with the same rehabilitative protocol and arrived at the same expected results.

\section{References}

1. Rizzo M.C. Avaliação da Imunidade. Pediatria 1993;29(3).
2. Andrade F., Montesano A., Bastos M. O Sistema Imune. Revisões Médicas Gerais, Jan-Mar 1994.

3. Thompson M. Genética Médica, $2^{a}$.edição, Rio de Janeiro, G. Koagan, 1993, 339pp.

4. Row I. Medicina Molecular. São Paulo, Roca, 1999, 226 pp.

5. Jorde L.Y.N.N.B. Genética Médica. 2a edição. Rio de Janeiro, Editora, 2000, 197 pp.

6. Carrión A. La inmunologia clínica actual. Rev Medica Chile 2000;128(6)

7. Carneiro S. Agamaglobulinemia congênita. Jornal de Pediatria R.J. nov-dez-1986.

8. Costa Carvalho B.T., Cocco R.R., Waldinei M.R., et al. Pneumonias de repetição em pacientes com deficiência de anticorpos e imunoglobulinas normais. J Pneumonologia 2002;28(3):155-8.

9. Márquez D.E. Extrahospitalay pneumonia: associated risk factors poor to the classification by knowing area in the Documentation Centers. Caracas 2003[49].

10. Calsaverini-Leal R. Manual clínico do fisioterapeuta pneumofuncional. Editora Santos: São Paulo, 2006.

11.Nakie C.M. Bronquiectasias. In: Razov T. [editor]. Doenças pulmonares em pediatria. Diagnostico e Tratamento. $1^{a}$ ed. São Paulo: Ed. Atheneu 1999, 254-62. 